\title{
Development and test logics for structural silicone bonding design and sizing
}

\author{
Anneliese Hagl
}

Received: 28 December 2015 / Accepted: 14 March 2016 / Published online: 19 April 2016

(C) Springer International Publishing Switzerland 2016

\begin{abstract}
The application of structural silicone bonding requires dedicated design procedures due to technical challenges such as high degree of incompressibility of silicone, low Young's modulus and non-linear material characteristics. Advanced bonding designs feature point-wise or line-type bonding geometries beyond the application range of ETAG 002 which is the European guideline for structural silicone glazing. No straight forward approach exists to extend the rules of ETAG 002 accordingly. In order to fill this gap, the following topics need to be addressed: (a) Silicone bonding material tests for the identification of elastic characteristics beyond ETAG 002, (b) Small sample tests for identification and quantification of failure mechanisms in addition to ETAG 002, (c) Structural mechanical analysis methods complementary to material and small sample tests, (d) Safety concept exploiting the above mentioned tests and analyses for ensuring adequate bonding performance. This paper outlines a comprehensive development approach answering this list in detail. Common material tests will be critically reviewed in view of fracture behaviour as small sample tests demonstrate a totally different failure mechanism compared to usually applied dog-bone or H-type sample tests. Complementary Finite Element Analyses based on material test characteristics allow correlating the test results with limit loading levels. These values
\end{abstract}

\footnotetext{
A. Hagl $(\varangle)$

A. Hagl Ingenieurgesellschaft mbH, Am Steinberg 34, 82237 Wörthsee, Germany

e-mail: hagl@a-hagl-ingenieure.de
}

serve for extrapolation from small sample test results to the envisaged full scale application. Within this extrapolation procedure, an adequate safety concept needs to be embedded accounting for temperature, humidity, aggressive environment etc. In this paper, a potential safety concept is presented for advanced line-type and point-wise bonding geometries.

Keywords Silicone - Bonding design - Structural Glazing · ETAG 002

\section{Introduction}

Nowadays, large glass elements and glass facades attract from aesthetic point of view of clients, architects and engineers in increasing manner. Advanced manufacturing and construction techniques and increasing knowledge how to engineer with this material, glass appears as ideal material for protecting humans on the one hand from unfriendly environmental conditions and for serving transparency on the other hand in order to provide natural light to the inhabitants. In addition, glass facades are often used as stylish elements for representative buildings.

The application of glass elements immediately poses the challenge to select an adequate connection technology. For classical glass facades, a simple bonding of rectangular cross section made by silicone material was already applied quite early in order to connect the glass elements with the underlying framework. 
For more advanced designs e.g. using glass fins, first choice in the past was the usage of screws and bolts as structural design elements ensuring the mechanical load path between glass elements and building. Drawbacks of this approach were:

- The point-wise introduction of loads leading to unfavourable stress concentrations

- The preparation of the glass elements with drilled holes and the hereby required accuracy (especially for laminated glass)

- The high number of parts asking for related logistics

During were developed in order to replace these connection elements for load carrying structures by advanced bonding designs. Figure 1 shows as example a comparison of a conventional design approach based on bolted point supports and the realized solution using a U-type bonding (Hagl 2003; Schadow 2006). It is obvious that this design offers a lot of advantages such as:

- An optically attractive solution due to the bonding hidden behind a façade profile

- Low manufacturing costs due to avoidance of drilling holes

- Simple assembly due to high accuracy of preassembled components, low number of parts and reduced danger of glass edge damaging due to the metallic edge protection

- More efficient material usage due to avoidance of stress concentrations leading to lower glass thickness

- Improved failure behaviour due to distributed support of the glass component by the bonding and the profile
Silicone proved to be a suitable bonding material for structural engineering due to its special characteristics. Its usage in bonding is also labelled as soft bonding due to its high flexibility evoked by low material stiffness, high strains and the suitability of large bonding thickness of several millimetres. Thus, it is well suited to compensate e.g. for thermal strains and for manufacturing tolerances compared to stiff bonding designs such as polyurethane etc. Other characteristics of silicones are:

- The resistance against weathering e.g. sun light, heat, ozone, $\mathrm{SO}_{2}$

- Low tendencies of aging such as stiffening or softening (Wolf and Cleland-Host 2004)

- Applicability for a wide temperature range $\left(-50^{\circ} \mathrm{C}\right.$ up to $+150^{\circ} \mathrm{C}$ )

- No oxidation at high temperatures

- Low absorption of humidity and related moisture expansion (swelling)

One reason for this special behaviour is related to the backbone of very stable $\mathrm{Si}-\mathrm{O}$ chemical bonding compared to classical $\mathrm{C}-\mathrm{C}$ backbones of usual rubber derivates (Habenicht 1997). From physical point of view, silicone shows the typical behaviour of hyper-elastic materials separating it clearly from classical behaviour of structural engineering materials such as concrete, steel and glass, see also Table 1. Hyper-elasticity means large elastic strains on the one hand and almost perfect incompressibility on the other hand (Treloar 2005). Furthermore, (filled) silicone shows typical rubber-like features such as the Mullins effect (Mullins 1948) i.e. softening after loading and visco-elasticity which might pose additional
Fig. 1 a and $\mathbf{b}$

Conventional versus bonding design for glass fin support (design study Herz-Jesu Church, Munich)

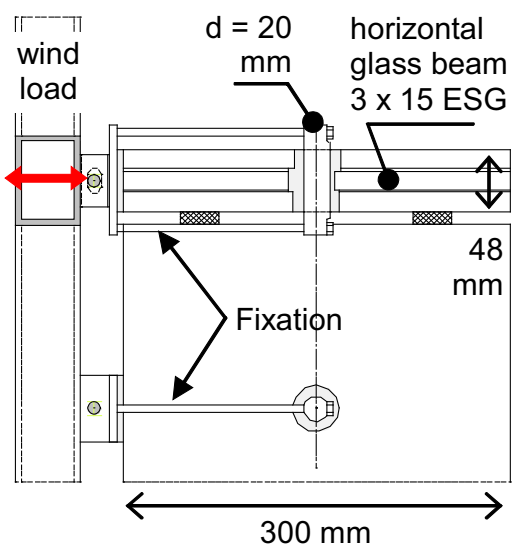

(a)

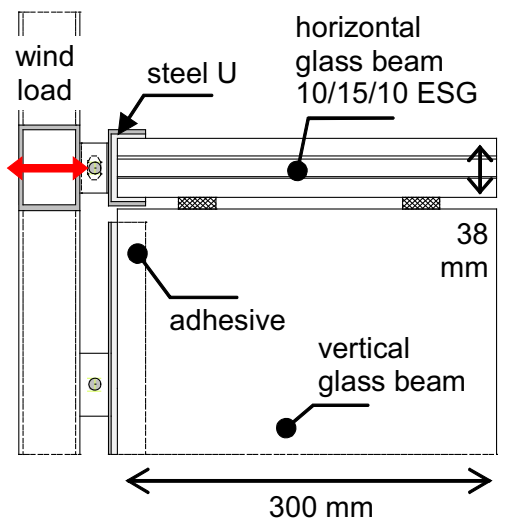

(b) 
Table 1 Mechanical properties of representative structural materials

\begin{tabular}{llllr}
\hline Material & Young's modulus $\left(\mathrm{N} / \mathrm{mm}^{2}\right)$ & Poisson's ratio & Density $\left(\mathrm{kg} / \mathrm{m}^{3}\right)$ & Thermal expansion $\left(10^{-6} / \mathrm{K}\right)$ \\
\hline Silicone & $1-2$ & $<0.5$ & 1330 & 190 \\
Glass & 70,000 & 0.23 & 2500 & 9 \\
Stainless steel & 170,000 & 0.3 & 7980 & 12 \\
Aluminium & 70,000 & 0.3 & 2700 & 24 \\
Concrete (C25/30) & 31,000 & $0.15-0.25$ & 2500 & 10 \\
\hline
\end{tabular}

challenges in design as material models for silicone might get quite complex (depending on the modelling objectives).

In order to simplify the design task with such a complex material, guidelines exist which trade simplicity of the design procedure with a very narrow design window. The ETAG 002 (EOTA 2001) is an example for such an approach balancing simple tests and engineering methods with tight design constraints. Hereby the innovative potential of bonding in structural engineering application is significantly limited. Focus in ETAG 002 is put on two-sided line type bonding designs of rectangular shape which can be considered as standard application of 'mass production' type for structural glazing of facades. Material performance is mainly taken into account by exploiting H-type samples which represent the bonding geometry and boundary conditions in small scale. Figure 2 shows the behaviour of silicone under tensile loading for both a conventional dog-bone specimen and the H-type small sample test of ETAG 002. As the presented properties are the engineering strains and engineering stresses (hereby eliminating different test lengths and cross section areas of the specimens), the results can directly be plotted in one figure. The H-type specimen typically features higher stiffness and lower limit stresses and strains than the dog-bone specimen under tensile loads. The test curves plotted in this figure are representative curves selected from a database consisting of more than 100 samples. The test results in this figure-as well as for all the other figures if not otherwise mentioned-are obtained at room temperature and approximately $50 \%$ relative humidity. Furthermore, representative curves are also used for the other figures.

The difference in behaviour between the two test articles can be easily explained referring to Table 1 . Typically the attachment plates of the H-type specimen are made of aluminium, steel or glass showing

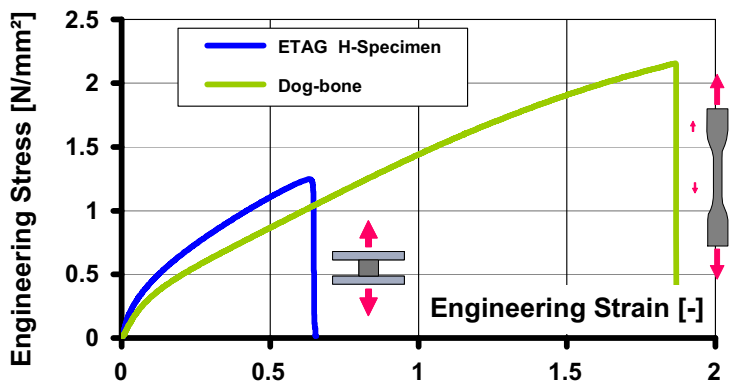

Fig. 2 Silicone tensile tests: Dog-bone versus ETAG002 H-type specimen

Young's moduli of several orders of magnitude higher than silicone. Thus, the silicone bonding in the H-type specimen can be considered as almost rigidly attached leading to different kinematics. While this very stiff attachment leads to significant suppression of lateral contraction of the silicone close to the interfaces, the dog-bone specimen allows the silicone to freely contract in lateral direction. In combination with the almost perfect incompressibility of silicone, the constraints in lateral contraction of the H-type specimen leads to higher effective stiffness as the H-type specimen can not trade elongation versus lateral contraction in the same manner as the dog-bone specimen considering the boundary condition of nearly constant volume.

On the other hand, the suppression of lateral contraction of the H-type specimen leads to non-uniform stress distributions and to stress concentrations allowing to explain the earlier failure with respect to engineering strains. In combination with higher stiffness, this property translates also into lower engineering stress values to be achieved for the H-type specimen. Concluding, the combination of typical structural engineering materials with silicone-as demonstrated by the H-type specimen by the attachments typically built of metals and/or glass_-offers the possibility to significantly 
Fig. 3 Comparison of mechanical characteristics for different silicone applications loaded by nominal stress $\sigma_{\mathrm{N}}=1 \mathrm{~N} / \mathrm{mm}^{2}$

\begin{tabular}{|c|c|c|c|c|}
\hline $\begin{array}{c}\text { Specimen } \\
\text { Type }\end{array}$ & $\begin{array}{c}\text { Dog-Bone } \\
\text { Specimen }\end{array}$ & $\begin{array}{c}\text { ETAG H-type } \\
\text { Specimen } \\
\begin{array}{c}\text { Stress and strain } \\
\text { field }\end{array}\end{array}$ & $\begin{array}{c}\text { Planar Round } \\
\text { Point Support }\end{array}$ & $\begin{array}{c}\text { U-type Point } \\
\text { Support } \\
\end{array}$ \\
\hline Strain $\varepsilon_{N}=\Delta / / l_{0}^{*}$ & 1,19 & 0,82 & 0,08 & 0,06 \\
\hline Stiffness $\sigma_{N} / \varepsilon_{N}$ & 0,84 & 1,21 & 12,9 & 16,9 \\
\hline $\begin{array}{c}\text { Stiffness related } \\
\text { to dog-bone }\end{array}$ & 1 & 1,44 & 15,4 & 20,1 \\
\hline
\end{tabular}

* Nominal stress $1 \mathrm{~N} / \mathrm{mm}^{2}=\sigma_{\mathrm{N}}$ influence the properties of the bonding by adequate design of the bonding geometry. This interesting feature allows tailoring mechanical characteristics of the bonding such as stiffness and even failure mechanisms to a large extent. This statement is underlined by the examples given in Fig. 3 in view of flexibility.

In Fig. 3, strains of different silicone applications are compared for a nominal loading of $1 \mathrm{~N} / \mathrm{mm}^{2}$ for a representative dual component structural glazing silicone. Based on the strain values, a secant stiffness is derived for a dog-bone specimen, for the H-type specimen of ETAG 002, for a planar point support and for a U-type point support. The stiffness properties of the latter three assemblies is referred to the dog-bone one showing that for these applications, the stiffness of the bonding can be easily varied beyond one order of magnitude. As will be shown later, also the failure mechanisms of these applications differ e.g. due to dual load paths in case of the U-type point support. The flange length is here the key parameter for partitioning into a tension load path and a shear load path. It is obvious that for the adequate tailoring of the bonding properties, a more detailed knowledge of the silicone material is required compared to the more pragmatic approach followed by ETAG 002. The paper addresses this challenge by the following sections:

- Silicone bonding material tests for the identification of elastic characteristics beyond ETAG 002

- Small sample tests for identification and quantification of failure mechanisms

- Structural mechanic analysis methods for silicone bondings
- Safety concept for ensuring adequate bonding performance

\section{Silicone bonding material tests for the identification of elastic characteristics beyond ETAG 002}

In order to describe the silicone material characteristics in bonding designs appropriately, two pre-requisites need to be fulfilled in advance. First, material laws need to be set up which describe the structural behaviour of the silicone material to an adequate extent from engineering point of view. Second, the parameters of these material laws need to be determined by results of adequate material experiments. In this section, focus is put on mechanical properties such as elasticity and fracture behaviour due to the special characteristics of the silicone material. Other physical properties such as thermal or humidity based extension of the test material might be identified by conventional tests as for other materials.

Regarding the elastic properties, material laws might describe time independent or time dependent behaviour (e.g. depending on strain rates). It is obvious that time independent behaviour is simpler to manage from both descriptive and experimental point of view as one external input i.e. the time dependent load history can be ignored. In view of material law application, it needs to be checked for this approach whether the sizing of the bonding is significantly affected by strain rates or not. For conventional loading of the silicone bonding 
e.g. by dead loads, thermal loads or wind loads the neglecting of load rates can be assumed for sizing purposes. For bomb blast linked to high strain rates this is surely not the case.

With respect to time independent material laws, linear elastic or hyper-elastic material laws might be suitable depending on the required strain amplitudes. A peculiarity of filled rubbers and silicones is the Mullins effect describing softening of the material characteristics after pre-loading. This behaviour can be interpreted as a kind of damaging, and modified hyper-elastic material laws were developed in order to describe this behaviour e.g. using a damage parameter. The Mullins effect should not be mixed up with visco-plastic behaviour where plastic strains remain after unloading of the material. For the description of the time dependent behaviour, visco-elastic properties need to be identified by time dependent loading e.g. by cyclic time histories of varying amplitudes and rates.

Concerning fracture behaviour, fatigue needs to be differentiated from typical quasi-static behaviour. As already outlined in Sect. 1, fracture behaviour of silicone might differ comparing pure material tests (e.g. dog-bone tests) and small sample tests (such as H-type specimens) e.g. evoked by non-uniform stress distributions. Figure 4 demonstrates the impact of bonding stress singularities at the edges of the bonding by a failure starting in these areas with high stress values. Thus the failure is triggered by the special design of the specimen not allowing any conclusions on the pure material behaviour itself by simple analysis means. In any case in this paper, it is assumed that silicone bondings mechanically degrade due to cohesive failure and not due to adhesive failure. This failure mechanism can typically be ensured by adequate surface treatment e.g. by the use of primers etc. in accordance to manufacturer instructions and guidelines.

For most of today's structural engineering applications, silicone behaviour can be approximated by a time independent description taking into account limit loads i.e. limit stresses or stiffness degradation limits for bonding sizing purposes. Thus, material tests are required to provide related material properties as silicone characteristics can significantly vary depending on the chemical ingredients and the production process. Furthermore, curing might be an important parameter especially for one component silicones curing by humidity.
The most basic description of isotropic elastic material-i.e. independent from load orientation-is given by three parameters of which two are independent and the third can be derived. These parameters are:

- The Young's modulus E describing the change of normal stress $\sigma$ due to change of normal strain $\varepsilon$ in laterally unconstraint conditions: $\sigma=\mathrm{E} \varepsilon$

- The shear modulus $\mathrm{G}$ describing the change of shear stress $\tau$ due to change of shear strain $\gamma: \tau=\mathrm{G} \gamma$

- The Poisson's ratio $\nu$ describing the lateral contraction $\varepsilon_{\mathrm{q}}$ due to longitudinal extension $\varepsilon_{1}: \nu=$ $-\varepsilon_{\mathrm{q}} / \varepsilon_{1}$.

The material properties $\mathrm{E}, \mathrm{G}$ and $\nu$ are linked by the equation $E=2 G(1+v)$. It should be highlighted here, that hyper-elastic material such as rubber and silicone can also be described by these linear elastic material laws for small strains. Thus, it can be directly concluded that at least two different kind of material tests are required to describe the elastic behaviour of silicones. Exploiting the assumption of incompressibility, one set of tests can be replaced by setting $\nu=0.5$.

Typical test set-ups for this purpose are tension and shear tests. For tension tests, dog-bone specimens are proven test geometries in order to identify material properties under uniaxial strain, see Fig. 5 left. Regarding post-processing of the experiment it has to be kept in mind that the displacement travel of the testing machine is not suitable as only the middle part of the specimen satisfies almost perfect uniaxial material loading. Thus, extra means are required to measure the displacements of the test range. As silicone material is very soft, optical techniques such as video extension-meters are applied in order not to affect the test results by mechanical devices leading to mechanical interactions. As already mentioned in Sect. 1, H-type specimens are

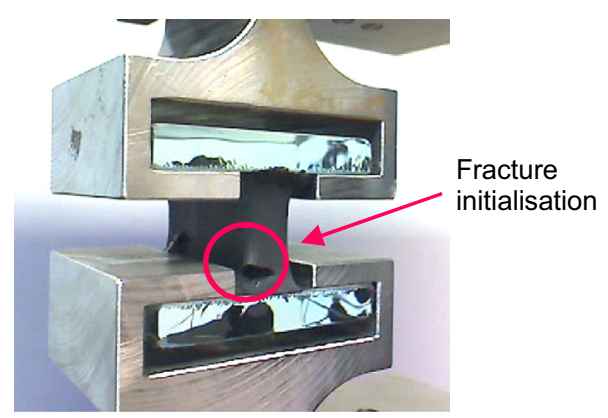

Fig. 4 H-type specimen starting to fail at bonding edge 


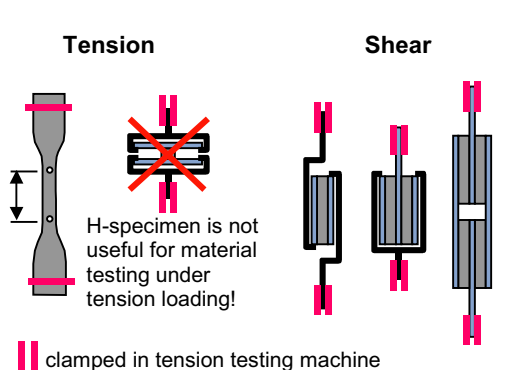

clamped in tension testing machine

Fig. 5 Typical test specimens used for silicone materials

not an alternative to these tests; they might be considered as complementary tests depending on the test objectives.

The samples discussed here are based on a standard 2-component structural glazing silicone of Dow Corning which is certified according to ETAG 002. The other tests in this paper refer to the same material.

Regarding shear tests, it is in general more difficult to achieve a pure shear state as a pure shear state is characterised by shear loads acting on all four interfaces in the shear plane. Typically a simple shear test is used instead as shown in Fig. 5 with respect to the three specimens in the middle. Simple shear tests are designed by two attachment plates which introduce relative displacements. Free warping as a kind of imperfection appears at the free surfaces at the ends of the specimen. In case the specimen is long compared to thick the area with the free warping can be neglected due to low impact. Nevertheless, rotation of the attachment plates might be a more important issue affecting the test results. Multiple specimens might eliminate this issue by trading the improved load introduction versus loss of quantitative insight during failure as the multiple bodies might fail differently. Figure 6 underlines this statement by showing the different pre- and post-failure characteristics of the specimens.

Specimen $2 \mathrm{xS}$ shows significant rotations which might also explain the different behaviour in the prefailure domain.

Silicone material shows an almost perfect incompressibility as typical characteristics of hyper elastic material behaviour. The level of incompressibility can be assessed by comparing the bulk modulus with the shear modulus. This behaviour is also described by the Poisson's ratio v. If the Poisson's ratio is approaching 0.5 from below it can be shown by simple analytical means that the material tends towards incompressible

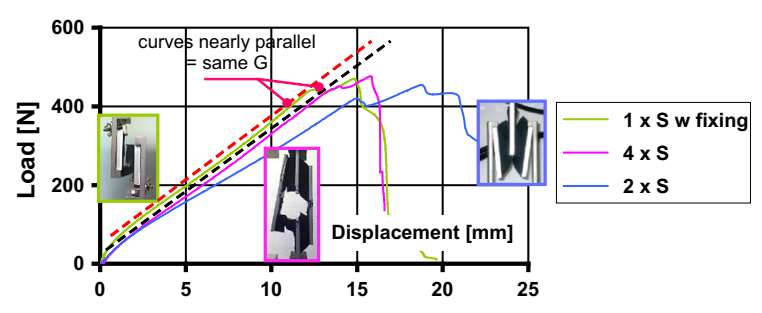

Fig. 6 Comparison of shear test specimens

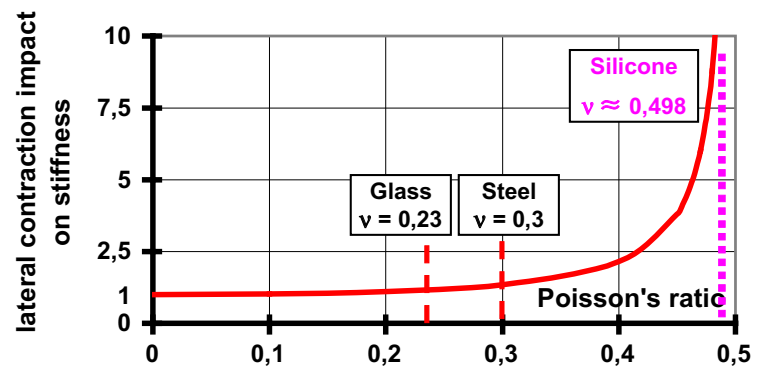

Fig. 7 Impact of Poisson's ratio on stiffness in case of perfect suppression of lateral contraction

behaviour. From a theoretical point of view, incompressibility leads to a decoupling of the hydrostatic pressure (or hydrostatic tension) from the displacement field meaning that hydrostatic stresses are not affected by deformations but by boundary conditions i.e. external loads. On the other hand, perfect blocking of lateral contraction leads to infinite stiffness for the incompressibility case as shown in Fig. 7. In this figure, the ratio of constraint to unconstraint stiffness is plotted versus Poisson's ratio.

An evaluation of the Poisson's ratio for a representative two-component silicone by optical means (bi-axial video extensometer measurements) by using the specimen geometry in Fig. 5 right, is demonstrated in Figs. 8 and 9 . Figure 8 shows the lateral strains $\varepsilon_{\mathrm{q}}$ plotted versus the longitudinal strains $\varepsilon_{1}$. In theory, the negative slope of the regression line provides the Poisson's ratio — here 0.4979—and the line should intersect the origin. This figure demonstrates very good agreement between theory and experimental data. On Fig. 9, the obtained Poisson's ratio is directly plotted versus longitudinal strain.

Perfect or almost perfect incompressibility has also other consequences with respect to constitutive material laws and several test approaches are no longer independent. Typical material testing scenarios are:

- Simple shear tests, see Fig. 10 


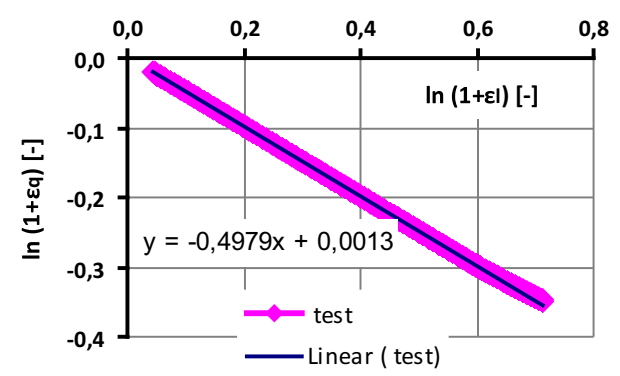

Fig. 8 Regression of lateral strains versus longitudinal strains

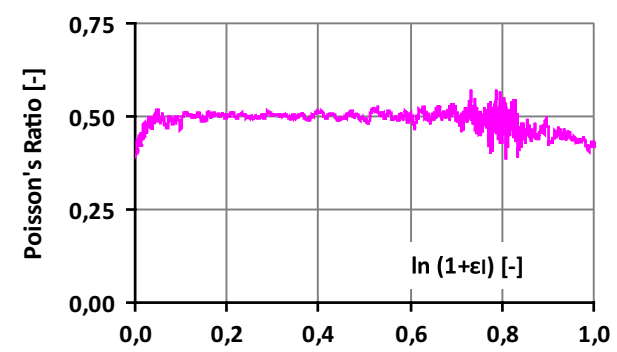

Fig. 9 Poisson's ratio versus longitudinal strain

- Compression tests, see Fig. 11

- One-axial tension or compression tests, see Fig. 12

- Bi-axial tension (or compression) tests, see Fig. 13

- Plain tension tests (also labelled as pure shear tests), see Fig. 14

Simple shear tests are typically characterised by large displacements and low stiffness for hyper-elastic material such as silicone as the molecular structure and the related properties of this kind of polymers allows high mobility of the chain molecules and thus low resistance against external deformations. As already mentioned, care has to be given to specimen design in order to reduce warping phenomena violating perfect shear conditions as much as possible.

Different to shear tests, compression tests typically reveal orders of magnitude larger stiffness values compared to the shear tests. The molecular explanation for this behaviour is seen in the high energy required in order to change the length of the molecule chains. It should be added that it is quite difficult from practical point of view to design adequate test set-ups for pure compressive (or tension) loading. Thus other tests were defined in order to quantify the Poisson's ratio and thus compressibility. One approach is shown in Fig. 5 right where the Poisson's ratio is determined by sensing the lateral contraction by optical means such
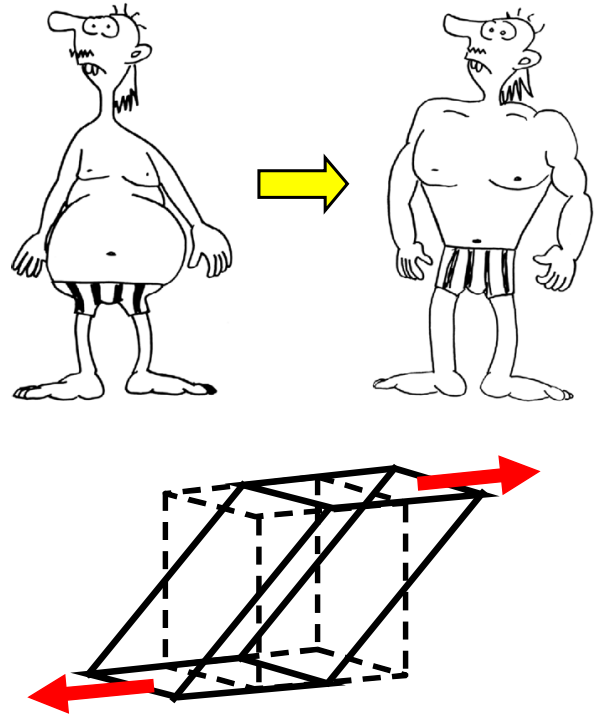

Fig. 10 Simple shear test

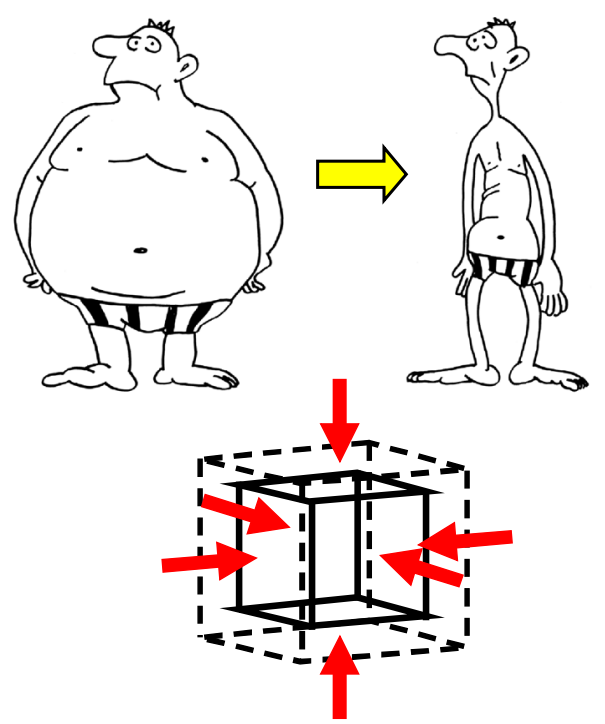

Fig. 11 Compression test

as video extensometers. Measurement accuracy might be an issue here as the fundamental change of material behaviour approaching $\nu=0.5$ is not adequately scaled by measuring geometric parameters. An alternate approach is described in (Wolf and Descamps 2002) exploiting the characteristics of wave propagation in the silicone material.

In Fig. 12, a tension test set-up is shown. For perfect incompressibility, same test results are achieved by stretching the specimen uniaxial as shown on the left in 


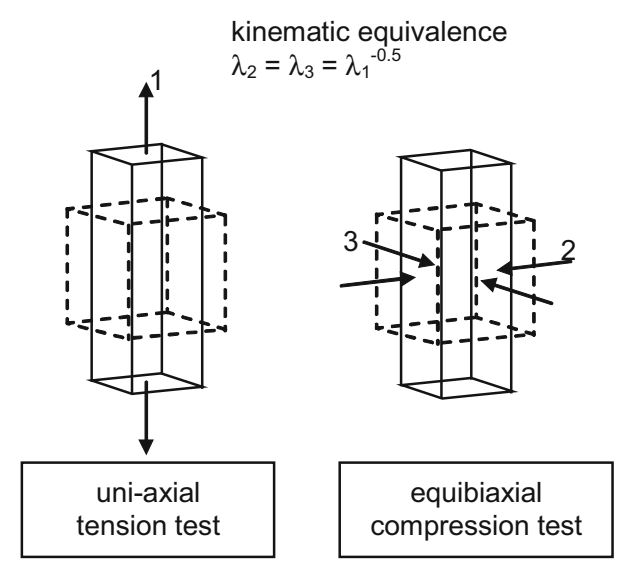

Fig. 12 Tension test

direction 1 or by compressing lateral directions 2 and 3 as shown on the right. The stretch $\lambda$ is hereby defined by the deformed specimen length divided by the undeformed length. The difference from material point of view is only the hydrostatic pressure while the deformation patterns are the same. Based on this outcome, a

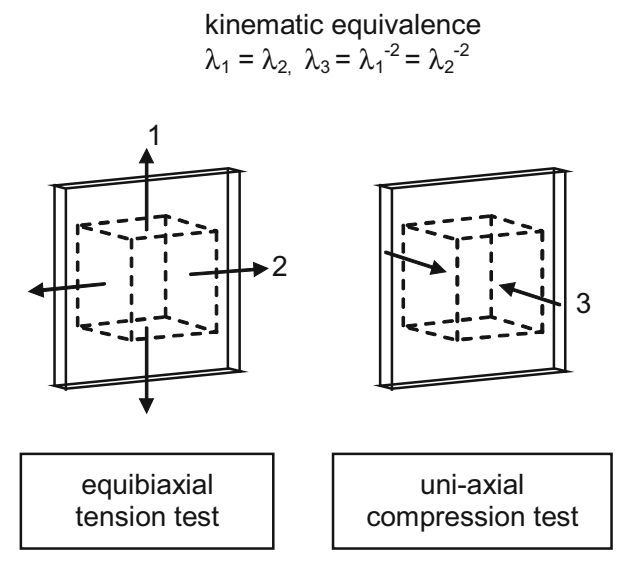

Fig. 13 Bi-axial tension test unique manifold of loading schemes exist to generate the motion patterns in the figure as load combinations of axial loading and lateral compression can be used for the same result. Of course, the statement is valid as well for uniaxial compression and lateral tension.

In a complementary manner to Fig. 12, duality is shown for bi-axial tension tests in directions 1 and 2 and uniaxial compression tests in direction 3 in Fig. 13. This duality is interesting in the sense that uniaxial compression tests are typically difficult to design and to perform due to potential buckling of the specimen leading to compact specimen designs and due to friction issues at the interfaces if placed between interfacing plates of the testing machine. Nevertheless, the adequate introduction of biaxial loading schemes into the specimen by mechanical components challenges the test set-up as well. Due to buckling issues, a biaxial compression test might be less practical. Here, a uniaxial tension test might be favoured instead.

If displacements of the specimen can be perfectly constraint in one direction, plain tension or compression tests can be performed, see Fig. 14. The interesting point of these tests is that due to incompressibility conditions, tension strains in one direction are coupled to compression strains in the other direction in such a way that pure shear motions are achieved under 45 deg orientation angle for small motions. Thus perfect shear conditions can be achieved by this test approach. As already mentioned, the challenge in designing the test set-up is the avoidance of motions and strains in the lateral direction 2 .

Regarding applicability and validity of the material tests, focus should be put on stress and/or strain ranges assumed to be relevant for the bonding designs. Figure 15 shows the load-displacement or respectively stress-strain curves for tension tests and shear tests. The tension test curve reveals a significant non-linearregressive-behaviour before failure while the shear

Fig. 14 Plain tension tests

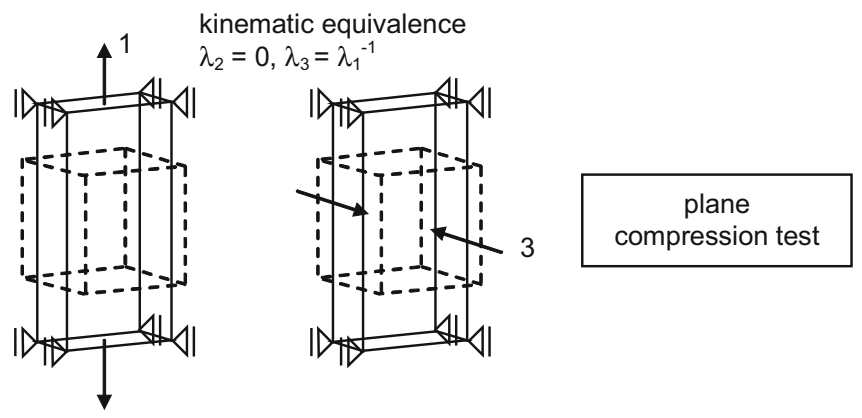


Fig. 15 Tension and shear load displacement curves
Tension

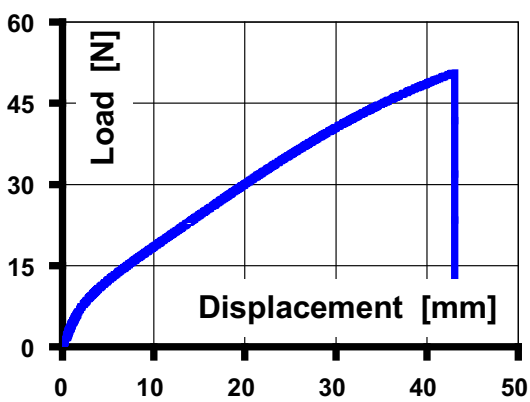

Shear

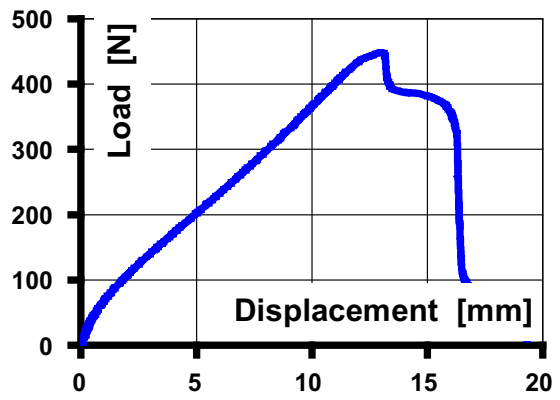

tests behave close to linear (or even slightly progressive) in the non-damaged region. Curvatures close to the origin need to be assessed in view of test imperfections as it typically proves to be quite difficult to perfectly insert a highly flexible specimen into the testing machine. It is obvious that non-linearity e.g. in the case of the tension curve can be easily considered in the tested range but extrapolation of the elastic material law is only adequately possible if based on physical first principles e.g. provided by material laws motivated by physical considerations.

It should be added here that the load rate has an impact on test results leading to stiffening and higher fracture loads for high rates which is e.g. of interest for bomb blast cases. The tests and test results discussed in this paper refer to quasi-static conditions and are extracted from material test standards, see (DIN 53504 2009). Displacement rates between $50 \mathrm{~mm} / \mathrm{min}$ and $500 \mathrm{~mm} / \mathrm{min}$ do not show significant impact on elastic behaviour for the related dog-bone specimens.

Of course, the stress-strain relationships obtained by the material tests which build the basis for deriving material laws depend on the environmental conditions. Especially in view of sustainability aspects, a variety of operating conditions might be of interest such as:

- Temperature variations

- Creep loading e.g. in view of dead loads

- Aged versus unaged conditions.

An example for the complex behaviour of silicone material is presented below in Fig. 16 plotting the characteristics under repeated loading of varying amplitude. The Mullins effect showing hysteresis characteristics is clearly visible. Interestingly, the material properties seem to be recovered if the amplitudes of the former load cycles are exceeded. This behaviour is also used in view of sizing as limit loads are linked

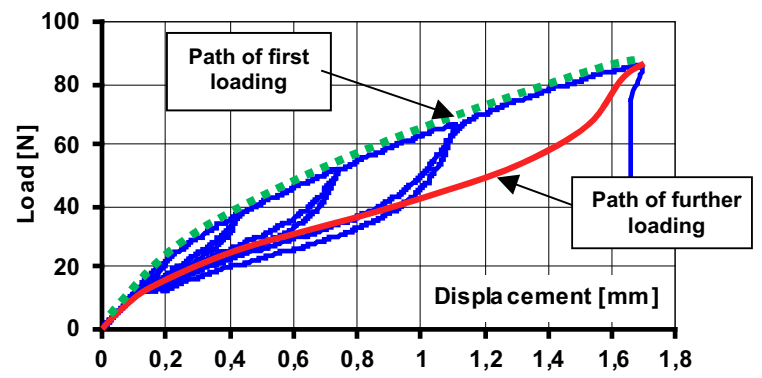

Fig. 16 Loading histories on material behaviour

to non-degraded material properties assuming a dominant i.e. critical load regime. On the other hand, care has to be given for the pre-treatment of specimens as in case of miss-treatment, in-voluntary softening of the specimen might occur as already mentioned above.

\section{Small sample tests for identification and quantification of failure mechanisms in addition to ETAG 002}

In this paper the topic "small sample tests" covers test segments of line-type bonding designs on the one hand and single point supports on the other hand. The most popular non-trivial line-type bonding design consists in a U-type bonding geometry built by attaching a (PFC) U-type element to the edge of a glass element. Other designs might be L-type bonding geometries in which only one of the flanges exist or T-type bonding geometries for which the inner glass panes need to be staggered. Concerning point supports, the most natural choice is to consider circular geometries due to rotational shapes with planar surfaces of the point supports leading to disk type bonding geometries (Wolf 2011).

Typically, the behaviour in shear for such kind of bondings can be described quite easily by mapping 
material test results of shear samples to the bonding geometry without experiencing too high deviations. In contrast, tension loading leads to a quite complex behaviour e.g. due to suppression of lateral contraction which does not occur for shear deformations. Going into the other direction, it is widely accepted based on engineering practice that elastomerics do not fail under high compressive loads in view of conventional structural engineering applications. Thus, focus is put on bonding behaviour under tension loading in this section.

\subsection{Line type bonding designs}

For line type bonding samples, the question arises which sample width to be used. From practical point of view, the testing machine and the implemented load cell capacity pose constraints towards the upper bounds of the small sample widths. On the other hand, the almost perfect incompressibility of silicone requests large widths in order to be representative especially for those bonding geometries featuring large constraint interfaces and low amount of free surfaces. In this case, the incompressibility leads to significant lateral contraction in case of low small sample widths hereby significantly altering mechanical performance of the bonding. Thus, a lower bound is given by representativeness of the line-type bonding with respect to limit loads and other characteristics. Specimen widths typically chosen in practice are in the order of $50 \mathrm{~mm}$ in order to use testing machines typically available in laboratories. A typical result for such kind of specimen is given in Fig. 17 in terms of a load curve plotted versus displacements for a representative two component structural glazing silicone. It is obvious that the pattern of this curve is quite different from the results obtained by a simple dog-bone specimen where a monotonous increase is terminated by a sudden collapse.

The behaviour of this complex bonding allows to exploit silicone bondings in a favourable way as the sudden failure event of dog-bone specimens poses serious engineering challenges by its aggressive failure pattern due to unstable crack growth (as it is e.g. the case for brittle ceramics) while this "yield-like" behaviour of the U-type bonding leads to good-natured failure behaviour. Regarding the U-type bonding, different segments of behaviour are visible which might be attributed to different mechanisms in the specimen (Hagl 2008a, b).

Initially, the bonding is fully operational and due to the high degree of encapsulation of the bonding in combination with the high level of incompressibility, the bonding reacts quite stiff which is in clear contrast to the low stiffness obtained by dog-bone specimens. This phenomenon can be localized at the front region of the U-type bonding geometry featuring dominant tensile loads. With increasing displacements, the slope of the load curve reduces indicating a change in bonding behaviour. In Fig. 17 this behaviour starts at approximately $2 \mathrm{~mm}$ displacement. The optical observation of specimen during testing and numerical analyses show that due to the high stiffness of the front region compared to the side regions bonding stresses increase quite significantly in this area initiating a material degradation at the front region while the side regions are still operative. At approximately $8.5 \mathrm{~mm}$ a sudden drop of loads is noted for the specimen presented in Fig. 17. This event is linked to the total failure of the front region combined with a redistribution of the main load path towards the side regions. The side regions show the characteristic motion pattern of shear which is typically related to soft behaviour and large displacements before break of the silicone. Thus, loads can still be
Fig. 17 Loads versus displacements for a U-type bonding under tension loading

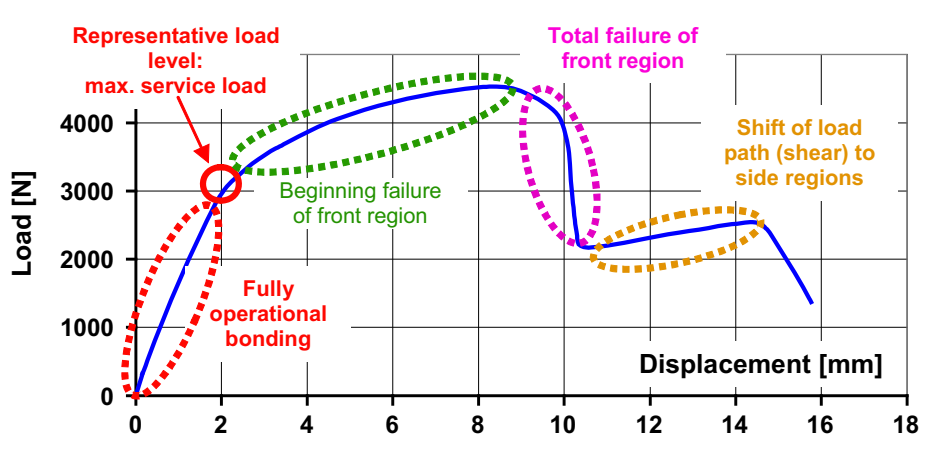

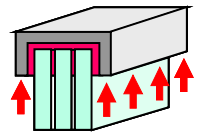

$3 \times 12 \mathrm{~mm}$ length $50 \mathrm{~mm}$ 
transmitted by this second load path of the side regions indicated also by a positive slope between 10.5 and $14.5 \mathrm{~mm}$. Beyond $14.5 \mathrm{~mm}$, total failure appears with degradation of the side regions.

This hypothesis was validated testing degraded Utype bondings in comparison to non-degraded (baseline) samples. Specimens were manufactured using PTFE foils applied on either the front region or the side region in order to disable load transfers in the related regions, see (Hagl 2008a). It could be shown that the dominant role of the front region for small displacements is clearly visible comparing the baseline bonding with the bonding with side regions disabled both featuring an operational front region in the beginning and high initial stiffness. On the other hand the behaviour at large displacements is easily identified as dominated by shear comparing the baseline bonding with the one with the front region disabled.

Similar results are obtained for T-type and L-type bonding geometries as presented and discussed in the same reference. All these bonding geometries in common are front and side regions reacting by tension and shear loads and the activation of front region(s) stiffening by suppression of lateral contraction.

This knowledge can be used to tailor the failure mechanisms of the U-type bonding. Small bonding thickness leads to stiffer behaviour for the front region (subjected to tension loading) and the side region (experiencing shear loading) as strains are higher for same displacements. Thus the slopes of the load curve can be modified by the adequate choice of bonding thickness for front and side regions. The thickness of the shear loads is obtained by the geometric combination of the PFC (parallel flange channels) width and the glass thickness while the thickness of the front region can be adjusted using spacers or similar devices. Nevertheless, one is not totally free in select- ing these bonding properties as manufacturer recommendations need to be followed asking for minimum and maximum bonding thickness e.g. in view of curing processes.

Load carrying capacities are affected by the bonding interface areas at front and side region as larger areas lead to higher limit loads. As the front region geometry is given by the thickness of the glass the related area needs to be considered as externally defined. On the other hand, the side region area can be designed by the length of the flanges of the PFC. Thus failure of the second load path i.e. at $14.5 \mathrm{~mm}$ in Fig. 17 can be quantitatively modified by this design means.

The test presented in Fig. 17 is performed by introducing the loading of the testing machine by brackets acting on the edges of the PFC. On the one hand this approach guarantees a quite homogeneous load introduction; on the other hand designs in structural engineering applications will be different using discrete anchor points such as bolts or similar. Typically, load introduction means are applied to the front side of the PFC/bonding thus potentially affecting mechanical performance of the front region by more inhomogeneous loading patterns.

\subsection{Point support bonding designs}

Point supports typically differ from the line type bondings discussed in the section before that only a "front region" of the bonding exists. This difference is also visible in the load curve, see Fig. 18.

In this figure, three regions with different mechanical characteristics can be identified. The first region ranging from zero to approximately $0.3 \mathrm{~mm}$ is characterized by large slope i.e. high stiffness. This behaviour is comparable to the results obtained for the U-
Fig. 18 Loads versus displacements for a planar point support under tension loading

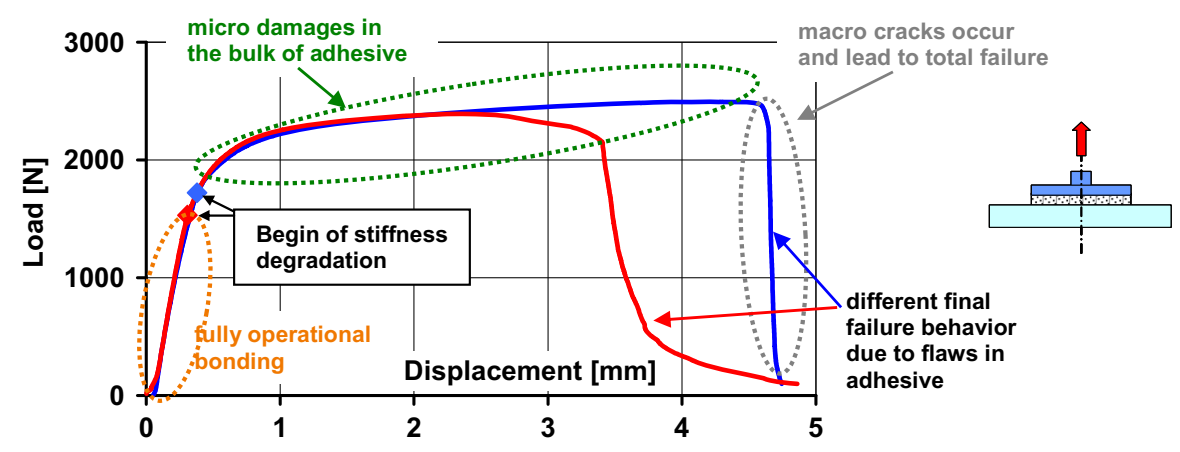




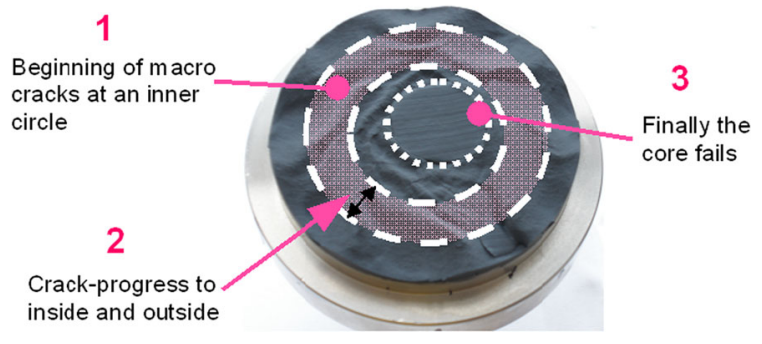

Fig. 19 Beginning fracture pattern for planar point support under tension loading

type bondings, see Fig. 17. The second region ranging from 0.3 to 3 or $5 \mathrm{~mm}$ respectively is characterized by a very low slope with almost no load increase. Finally, total collapse of the specimen is noted. In the figure, the behaviour of two different samples is shown being identical up to displacements of $2 \mathrm{~mm}$. As identified from the fracture surface after inspection, flaws are assumed to favour early failure for one of the samples.

In order to investigate the behaviour in region 2 in more detail, load histories of specimens were stopped at different levels and the specimens cut for investigation. An example for the beginning failure pattern is shown in Fig. 19. Interestingly, the systematic analysis of these patterns leads to the result that the macroscopic bonding failure does not start outboard or in the middle but in radial locations between one third and two thirds of the specimen. With increasing displacements, the fracture area propagates in inboard and outboard directions. Typically, cracks get visible at the outer surface at the end of region 2/start of region 3 .

In order to identify the beginning degradation of the point support which is relevant for sizing-e.g. in view of sustaining service loads-point support stiffness is derived by numerically differentiating the load curve obtained by the testing machine, see Fig. 20 .

Consistent to Fig. 18, high stiffness is obtained for region 1 i.e. for lower displacements than $0.3 \mathrm{~mm}$ and a sudden reduction is visible for larger displacements. Thus a bonding degradation on microscopic level is postulated for approximately $0.3 \mathrm{~mm}$. For the planar point supports of $50 \mathrm{~mm}$ diameter and $5 \mathrm{~mm}$ bonding thickness the degradation is linked to a level of approximately $1700 \mathrm{~N}$ for the investigated two-component structural glazing silicone. As expected, larger point support diameters lead to higher loads as for $70 \mathrm{~mm}$ diameter, load levels of $4100 \mathrm{~N}$ are obtained for the

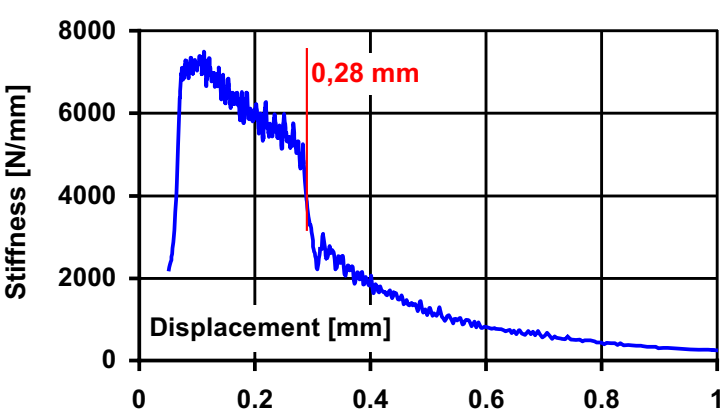

Fig. 20 Stiffness characteristics of planar point supports under tension loading

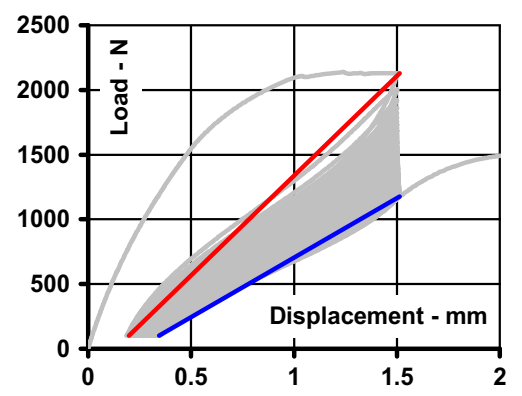

Fig. 21 Tension loading of planar point supports

same condition. For comparison, limit loads i.e. highest loads are noted as $2500 \mathrm{~N}$ for $50 \mathrm{~mm}$ and $5100 \mathrm{~N}$ for $70 \mathrm{~mm}$, see (Hagl 2008b).

Different attempts were taken to optimize the mechanical behaviour of point supports under tension loading. Convex and concave point support fittings were designed and tested, see also (Hagl 2007). The idea was to vary bonding thickness versus radius and to analyse the resulting mechanical behaviour. The experimental results show that concave point support shapes lead to slightly higher loads but less displacements in view of the total collapse. Thus potential for optimization seems limited in view of these tests. Other approaches are related to sunk point support specimens, see (Hagl 2008b). Here, dedicated pre-treatment of the glass units is required-i.e. drilling holes into the glass panes - which needs to be justified by improved performance (e.g. leading to lower number of point supports) or other cost saving issues.

The planar point supports were also tested under cyclic loading in order to check for potential fatigue issues. Swelling tension loads were applied by prescribed displacements, see Hagl (2010). In Fig. 21, maximum displacements for the load cycles were defined by $1.5 \mathrm{~mm}$ while minimum reversal conditions 


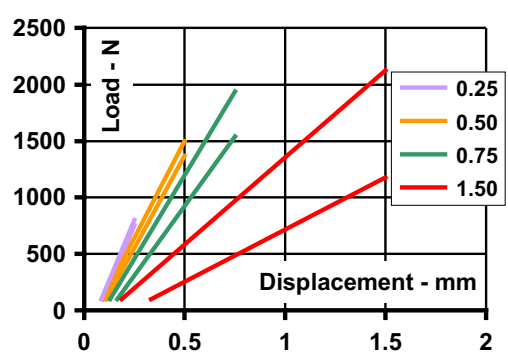

Fig. 22 Varying amplitude of tension cyclic loading for planar point supports

were given by a small tension load in order to avoid mixing up tension and compression load schemes. The Mullins effect is clearly visible by the first cycle showing high loads for increasing displacements while lower loads are obtained for decreasing displacements of the first cycle and for all subsequent cycles. Two lines are added to the figure in order to characterize this behaviour under cyclic loading using a kind of representative slopes. The red line connects the upper reversal point of the first cycle with the lower reversal point between first and second cycle while the blue line connects the related reversal points of the last tested cycle before breaking the specimen. For practical reasons i.e. testing time, the number of cycles was limited to 100 and related displacement rate was set to $1 \mathrm{~mm} / \mathrm{s}$. On the one hand, the number of cycles seem to be quite low with respect to conclusive statements in view of fatigue behaviour and resistance. On the other hand, changes are quite small approaching the last cycles.

Next, different displacement amplitudes were tested, see Fig. 22. The different amplitudes are represented here by the "slopes" of the first and last cycle. The increasing degradation is visible by comparing the last cycle slope with the first cycle slope showing larger slope reduction with increasing cycle amplitudes. The outcome of these tests indicate that a loading below 0.3 $\mathrm{mm}$ i.e. below stiffness degradation under quasi-static loading (Fig. 20) is also acceptable from fatigue point of view-at least with respect to the number of cycles performed during testing as results for 0.25 and $0.5 \mathrm{~mm}$ amplitudes indicate.

\section{Structural mechanical analysis methods for silicone bondings}

In order to improve physical insight in the structural mechanics of silicone bondings and in order to com- plement the experimental activities to understand the specimen behaviour in more detail, numerical studies are applied typically based on finite element methods (Gent 2001). For the adequate application of finite element analysis (FEA) a description of the material behaviour is required in terms of a material law for each part in the model. Material laws of interest for silicone might be:

1. Isotopic linear material description given by two material parameters (e.g. Young's modulus, Poisson's ratio)

This description is applicable for small and quasi-steady strains where non-linear behaviour and rate dependency can be neglected.

2. Hyper-elastic material laws based on parametric strain energy definitions

This description is applicable for large quasisteady strains where repeated loading and rate dependency can be neglected.

3. Hyper-elastic material laws including damage models

This description is applicable for large quasisteady strains under repeated loading where rate dependency can be neglected. Damage parameters are included to represent the Mullins phenomenon.

4. Visco-elastic material laws

This description is applicable for time dependent analyses in order to cover rate dependency of the silicone material characteristics.

The parameters of the chosen material laws need to be identified by material tests. It is important to note that the material model validity is only ensured to strain levels as considered for material parameter identification. Validity and accuracy of the material laws regarding extrapolation in terms of strains, strain rates and temperature is open and more or less directly linked to the nature of the material law, e.g. only descriptive/empirical suitable with focus on interpolation or based on physical principles allowing some kind of extrapolation.

Commercial FE packages provide automatic procedures to adapt the parameters of the selected material model based on provided specimen test results in 
a black box approach. Regarding this approach, validity of specimen test results has to be guaranteed, e.g. in view of the load curve origin in case of non-linear material behaviour, and typically no weighting between test points can be performed which might be useful to adjust the model to the load regimes of major interest. The automatic determination of the material constants is typically based on the following tests for distortional deformation constants:

- Simple tension/compression tests

- Equi-biaxial tension tests

- Simple shear tests

- Pure shear tests

For volumetric deformation constants, pure volumetric compression test results are required in principal.

In order to increase flexibility and to get more physical insight, a manual procedure is presented below starting with the exploitation of shear tests. The advantages and disadvantages of these two different methods can be concluded as follows:

- Automatic determination by related pre-processors of finite element packages:

- High user friendliness especially for novices

- Implementation errors reduced by automatic transfer of results

- No mathematical background required for optimization

- Manual determination as presented in this section

- High flexibility including and combining different experimental results

- Total control of optimization procedure inclusive weighting for different test points

- Consideration of imperfect test conditions by related parametric FE studies (taking into account imperfections, see e.g. Fig. 23)

Silicone behaves quite linear in a large range of shear offering the advantage of being quite insensitive in view of origin point issues and amplitudes of the load curves, see Fig. 24. The slope of the shear stress versus strain curve obtained by relating shear loads and shear displacements to the specimen geometry provides the shear modulus $G$. In combination with the Poisson's ratio $v$ this information is sufficient to define the isotropic linear material law. Young's modulus can be derived assuming isotropic linear behaviour by $\mathrm{E}=2 \mathrm{G}(1+v)$.

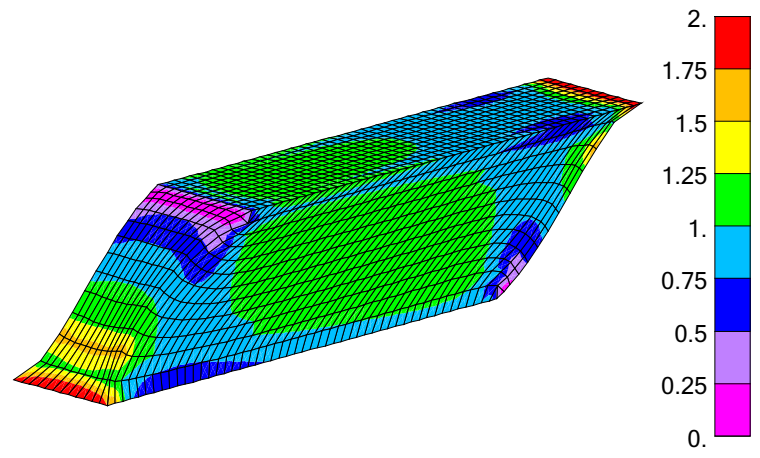

Fig. 23 FE analysis of ETAG 002 specimen under shear loading

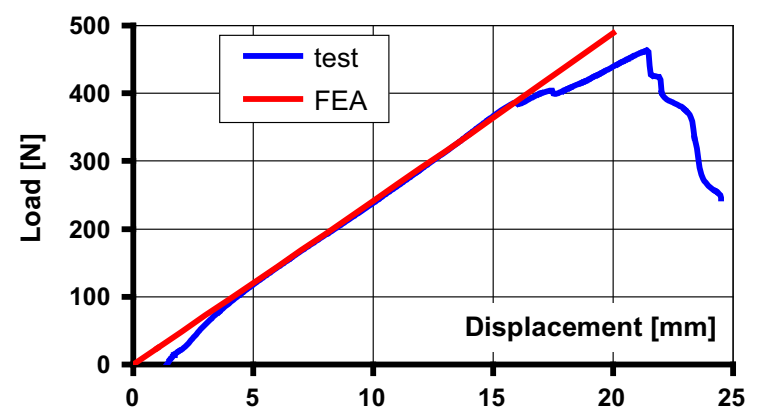

Fig. 24 Shear test results and comparison with FEA

In case the FEA supports perfectly incompressible material laws by special finite element formulations the Poisson's ratio can be set to 0.5 at first glance assuming perfect incompressibility of silicone. Alternatively, values from literature (Wolf and Descamps 2002) or derived by geometrical measurements as shown in Fig. 5 right can be applied. Parameter studies related to the Poisson's ratio might help to determine whether additional effort has to be spent on a more accurate Poisson's ratio definition. Furthermore, the application of Poisson's ratio values might be limited by numerical conditioning in case the formulation of the applied finite elements is not especially tailored towards the simulation of almost incompressible behaviour.

In order to check the correct implementation of the material law, it is recommended to model the specimen test also by FEA and to compare results as shown in Fig. 24. In this figure, experimental issues close to the origin are visible which are caused by imperfect placing the specimen in the testing machine. In case of inadequate manipulation of the specimen, the Mullin's effect might by visible for small loads i.e. close to the origin. The explicit exploitation of almost perfect 
linearity in shear compensates this issue. In addition the comparison allows to check for test imperfections such as warping on free surfaces as the FEA will feature this imperfections as well if adequately set up, see Fig. 23.

Typically tension tests by dog-bone specimens are available for the material descriptions. The load curves show declining characteristics at least for lower strains. Thus, these test results allow an adjustment of hyperelastic material laws. In this demonstration, a polynomial description for the strain energy density of hyperelastic behaviour is applied $\left(\mathrm{C}_{\mathrm{ij}}, \mathrm{D}_{\mathrm{k}}\right.$ material coefficients, $\mathrm{I}_{1}, \mathrm{I}_{2}, \mathrm{~J}$ invariants):

$W=\sum_{i+j}^{N} C_{i j}\left(\bar{I}_{1}-3\right)^{i}\left(\bar{I}_{2}-3\right)^{j}+\sum_{K=1}^{N} \frac{1}{D_{k}}(J-1)^{2 k}$.

In case of a low order material model featuring only two coefficients $\mathrm{C}_{10}$ and $\mathrm{C}_{01}$, the model is labelled Mooney-Rivlin. It can be shown by analytical means that for polynomial descriptions, the following statement used as boundary condition for parameter identification is valid for small strains: $\mathrm{C}_{10}+\mathrm{C}_{01}=\mathrm{G} / 2$. Thus, the objective to match the experimental results can be mapped to an adjustment of the two coefficients using "test" functions $\mathrm{f}_{10}$ and $\mathrm{f}_{01}$ which were obtained for $\mathrm{C}_{10}=\mathrm{G} / 2, \mathrm{C}_{01}=0$ and $\mathrm{C}_{10}=0$, $\mathrm{C}_{01}=\mathrm{G} / 2$. For this purpose a linear combination $\mathrm{y}^{\mathrm{fea}}$ of the "test" curves is set up using the scales $\alpha$ and $1-\alpha$ for the two test curves. Here, the special approach of the strain energy function consisting of a sum of terms is exploited for a given deformation pattern by adjusting the contributions of the various terms. This procedure is sketched in Fig. 25 by showing the test curve and these two numerical functions. A classical approach for the determination of the unknown coefficients consists in building the squared sum of differences between test and analysis and to minimize this sum using non-linear solvers capabilities in spreadsheet programs by asking for a minimum varying the coefficients $\mathrm{C}_{10}$ and $\mathrm{C}_{01}$ by the scale $\alpha$.

This approach is formalized by the following steps, see also Fig. 26:

1. Calculation of difference between numerical and experimental forces/stresses of the collocation points for initial selection of $\mathrm{C}_{10}$ and $\mathrm{C}_{01}$ :

$$
\Delta_{\mathrm{i}}=\mathrm{y}_{\mathrm{i}}^{\mathrm{exp}}-\mathrm{y}_{\mathrm{i}}^{\text {fea }}
$$

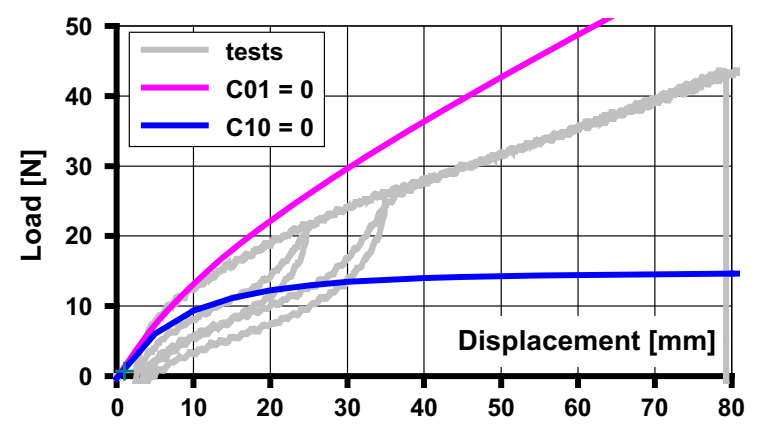

Fig. 25 Tension test curve and comparison and "test" curves $\mathrm{f}_{10}$ and $\mathrm{f}_{01}$

2. Weighting of test points if required (e.g. low weights for test points not trust worthy - for example Mullins effect on low strain area - or high weights for test points comparable to target loading asking for high accuracy):

$\Delta_{\mathrm{i}}^{\mathrm{w}}=\mathrm{w}_{\mathrm{i}} \Delta_{\mathrm{i}}$

3. Summing up of the square of all differences:

$\Delta=\Sigma\left(\Delta_{\mathrm{i}}^{\mathrm{w}}\right)^{2}$

4. Minimize sum $\Delta$ by adjusting coefficients $\mathrm{C}_{10}$ and $\mathrm{C}_{01}$ : In case of Mooney-Rivlin the optimal value $\alpha$ leads to $\mathrm{C}_{10}=\alpha / 2 \mathrm{G}$ and $\mathrm{C}_{01}=(1-\alpha) / 2 \mathrm{G}$.

The following remarks are added here for completeness:

- The Neo-Hook material law is based on $\mathrm{C}_{10}$ only which can directly be derived from the shear tests by $\mathrm{C}_{10}=\mathrm{G} / 2$. It should be noted that the Neo-Hook law might be applied for higher strain levels than the isotropic linear elastic material law but lower strain levels compared to Mooney-Rivlin.

- By enriching the test functions with higher order terms, hyper-elastic strain functions of higher order can be derived by the same approach e.g. modelling also turning points if required for high strain levels. Nevertheless, it should be highlighted that material models should be on the one hand as accurate as required in view of the applications but on the other hand as simple as possible in view of numerical stability. The most complex material model is not always the best.

- Boundary conditions might be added for the optimal coefficients $C_{i j}$ e.g. by asking for $C_{i j} \geq 0$. This condition guarantees stability of the material law by relating higher strains to higher strain energies as it is known for elastic material as basic principle. 
Fig. 26 Spreadsheet screen shot for determination of coefficients for Mooney-Rivlin

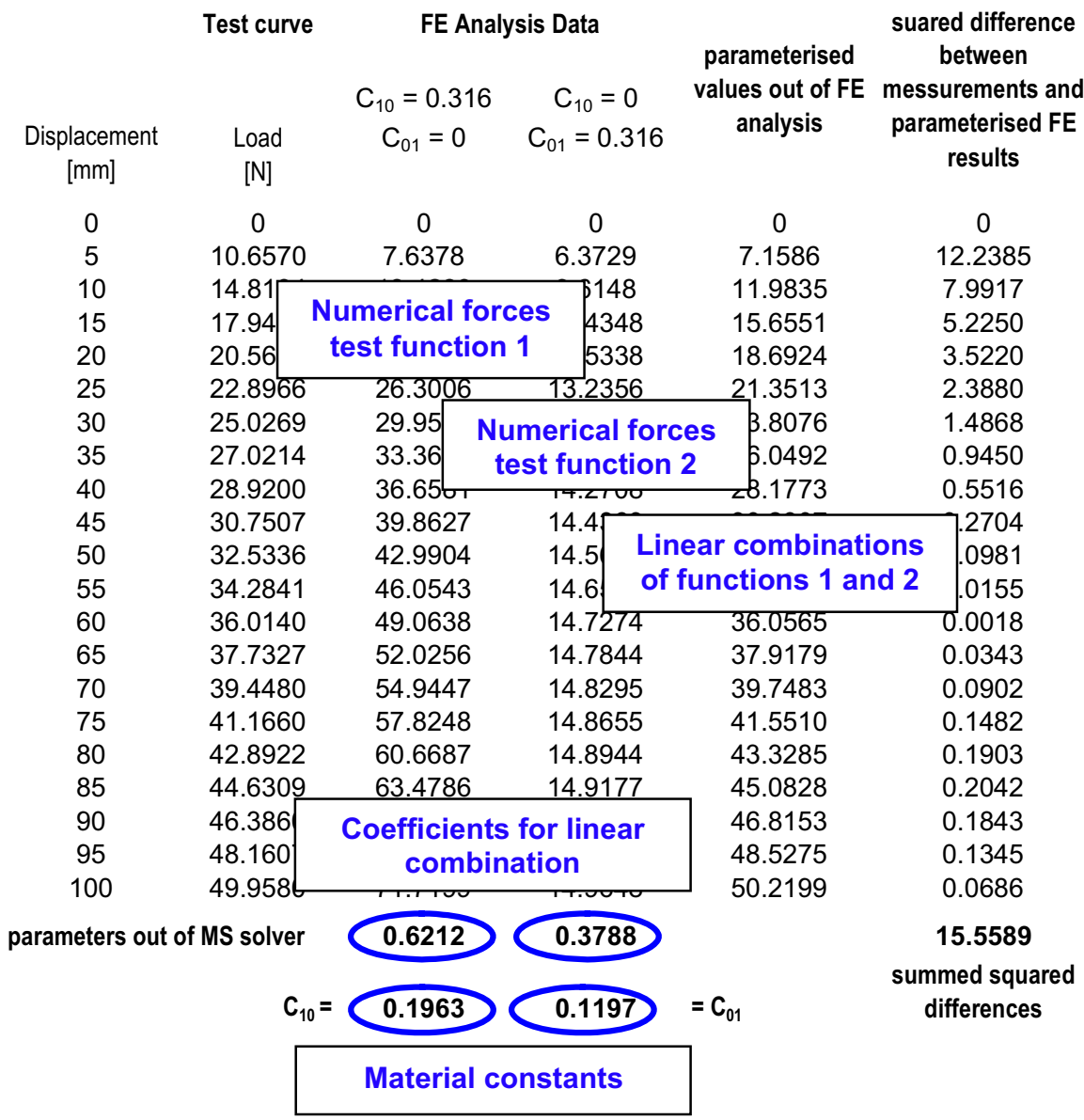

- This approach can also be applied in modified form for damage models in order to consider the Mullins phenomenon and for visco-elastic models in view of strain rate dependencies. Nevertheless, the underlying experimental data shall allow for the identification of these phenomena.

The identified material model can now be applied to the analysis of complex bonding designs such as the bonded point support in Fig. 27. The obtained strain and stress distributions within the silicone bonding allow the assessment of the loading of the bonding in view of service and limit load levels.

\section{Safety concept exploiting the above mentioned tests and analyses for ensuring adequate bonding performance}

The safety concept of the guideline ETAG 002 is based on quite high safety factors in order to compensate for simplifications see also (Pröbster 2013). In view of the most relevant load cases the safety factors are defined as follows:

- Short duration tension loading e.g. wind loads: The allowable stress level is set to $1 / 6$ of the $5 \%$ fractile value of the fracture strength $\mathrm{R}_{\mathrm{U}, 5} / 6$.

- Short duration shear loading e.g. thermal loads: The allowable strain level is set to $1 / 8$ of the maximum fracture strains.

- Long duration shear loading e.g. dead loads: The allowable strain level is set to $1 / 10$ of the short duration value as defined one step before.

The related experimental database refers to H-type specimens showing differences to dog-bone material tests with respect to tension loading as discussed in Sect. 2. Thus, this approach is affected by the given geometry of the specimen (e.g. width and length) as failure is triggered by stress concentrations at the specimen edges, see Fig. 28. For representative two- 
Fig. 27 FE Analysis of bonded point support
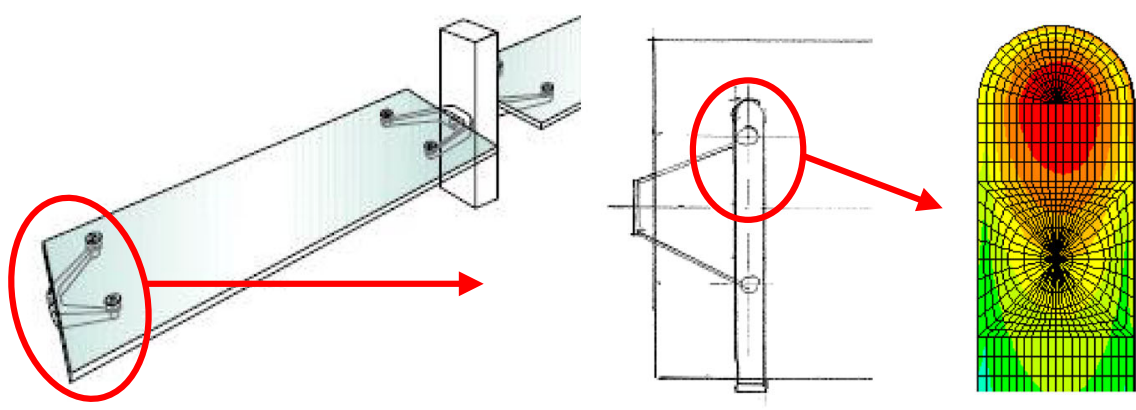
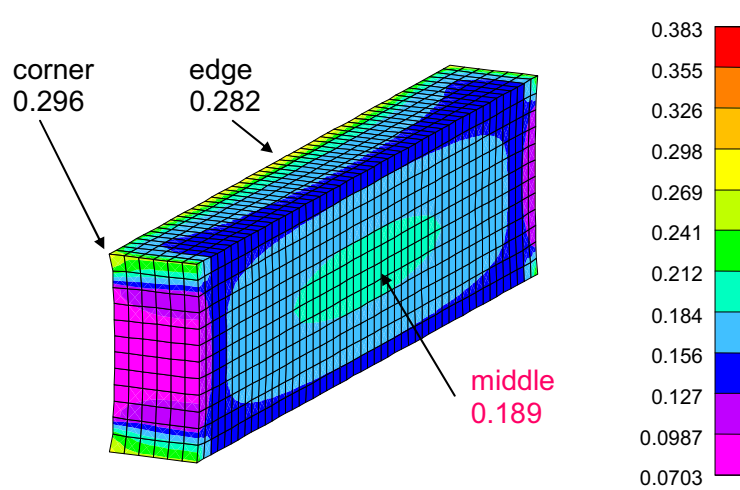

Fig. 28 Half model of ETAG 002 H-type specimen under tension loading, max. principal stress distribution $\left(\mathrm{N} / \mathrm{mm}^{2}\right)$

component structural glazing silicones these requirements translate to the following numbers for the design stress levels:

- Short duration tension loading: $\sigma_{\text {des }}=0.14 \mathrm{MPa}$ $=0.14 \mathrm{~N} / \mathrm{mm}^{2}$

- Short duration shear loading: $\tau_{\text {des }}=0.11 \mathrm{MPa}=$ $0.11 \mathrm{~N} / \mathrm{mm}^{2}$

- Long duration shear loading: $\tau_{\infty}=0.011 \mathrm{MPa}=$ $0.011 \mathrm{~N} / \mathrm{mm}^{2}$

For bondings in general, several load cases might be relevant for sizing. In principle, loads can be introduced at discrete attachment points (e.g. point support units or bolt connections of PFC) by forces or moments in all three spatial directions as shown in Fig. 29. Typically attachment design is performed in such a way that moments e.g. due to force offsets are minimized leading to forces as relevant design parameters. In addition thermal strains shall not be neglected as the different construction materials typically feature different thermal expansion. Regarding Fig. 29 tension, compression, lateral and longitudinal shear need to be considered. For a planar point support, this list is reduced to

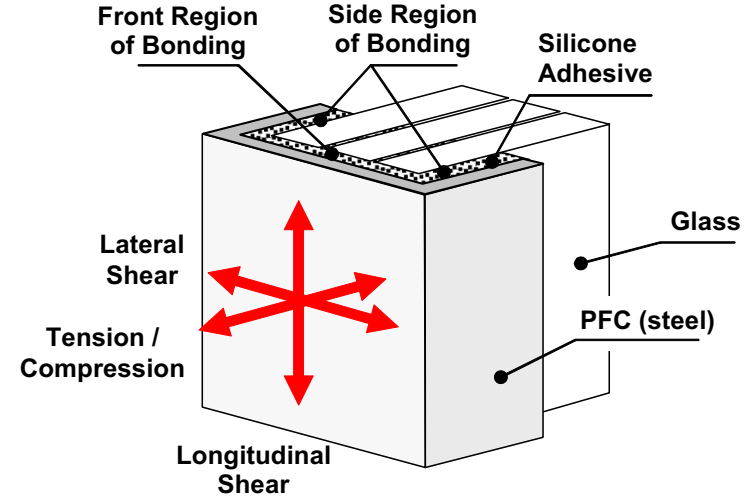

Fig. 29 U-type bonding and load scenarios

tension, compression and (longitudinal) shear due to axi-symmetric conditions.

As already mentioned, tension loading of silicone bondings is typically the most critical loading of the bonding material. In contrast, compression loading is not sizing for the bonding design as the silicone material is quite robust in view of loading in this direction. The lateral shear is assumed to be stabilised by the region loaded by compression i.e. by one of the side regions. Of course, sizing of the PFC needs to withstand this load case. Regarding longitudinal shear, the bonding is loaded by shear only and material characteristics obtained by shear tests can directly be applied e.g. in accordance to the approach given by ETAG 002 . This kind of loading leads to a quite soft reaction of the silicone bonding which is typically desired as thermal strains can be covered by shear. It should be highlighted in this context that in ETAG 002, bonding thickness is sized by allowable shear strains evoked by thermal displacements. This approach can also be mapped to complex bondings which react soft in the related direction. Thus, it is the tension load scheme which asks for special treatment for complex bonding geometries. 
Fig. 30 Maximum principal stress distributions for point supports at begin of degradation

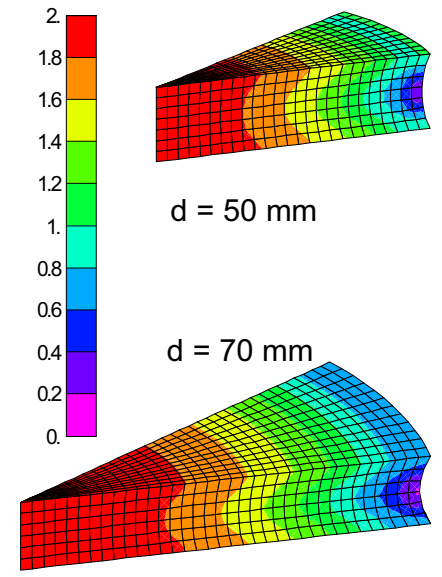

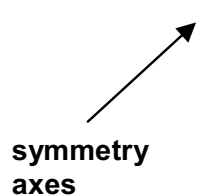

axes

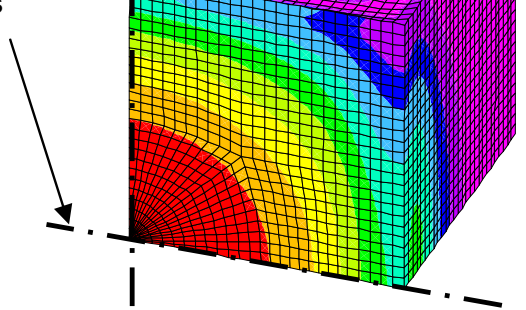

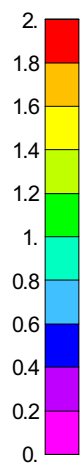

Fig. 31 Maximum principal stress distributions for small sample U-type specimen at begin of degradation

In view of complex bondings such as point supports and U-type bondings, small sample tension tests provide insight into the load carrying capacities of these bonding geometries. Figures 30 and 31 show that at the beginning of degradation, maximum principal stresses of approximately $2 \mathrm{~N} / \mathrm{mm}^{2}$ are obtained for the twocomponent structural glazing silicone. These maximum principal stresses are located in the middle of the specimens where encapsulation of the material is most effective leading to stress maxima in combination with the suppression of lateral contraction. It should be added that the usage of engineering strains is not appropriate in this case e.g. due to non-uniformity of stress distributions.

As the small sample specimen tests are typically performed under laboratory conditions (i.e. room temperature, humidity), additional knowledge with respect to material behaviour is required for safe design. Thus, knowledge of the silicone bonding by conventional testing is limited to:

- Temperature: typically room temperature

- Aging: typically unaged specimens

- Creep: typically short term load histories

Therefore additional tension and shear tests of the silicone material were performed in order to investigate the impact of these environmental conditions on silicone performance, see (FHM 2007). Artificial aging is related to the following procedure:

- 3 days @80 temperature

- 10 days@ $95^{\circ} \mathrm{C}$ temperature in de-mineralized water with cleaning agent $(5 \%)$

- 3 days @80 temperature

- 10 days@ $95^{\circ} \mathrm{C}$ temperature in de-mineralized water with cleaning agent $(5 \%)$ plus UV radiation $50+/-5 \mathrm{~W} / \mathrm{mm}^{2}$

- 1 day @ $23^{\circ}$ temperature

- 8 days @ $45^{\circ} \mathrm{C}$ temperature in salted water $(50 \mathrm{~g} / \mathrm{l}$ salt) plus UV radiation $50+/-5 \mathrm{~W} / \mathrm{mm}^{2}$

- 2 days @-30 ${ }^{\circ} \mathrm{C}$ temperature

- 1 day @ $23^{\circ} \mathrm{C}$ temperature $\rightarrow$ test performance

Specimens dedicated to creep were conditioned with a tension loading equivalent to $20 \%$ maximum strain for duration of 105 days. Figure 32 shows the dependency of fracture loads to temperature, aging and creep of the specimens. The bars plot the mean values while the error bars show the variations of the individual samples. The following conclusions can be drawn from this study, see also (Hagl 2008a):

- Higher temperature shows slightly lower limit loads while lower temperature leads to an increase of these loads. 


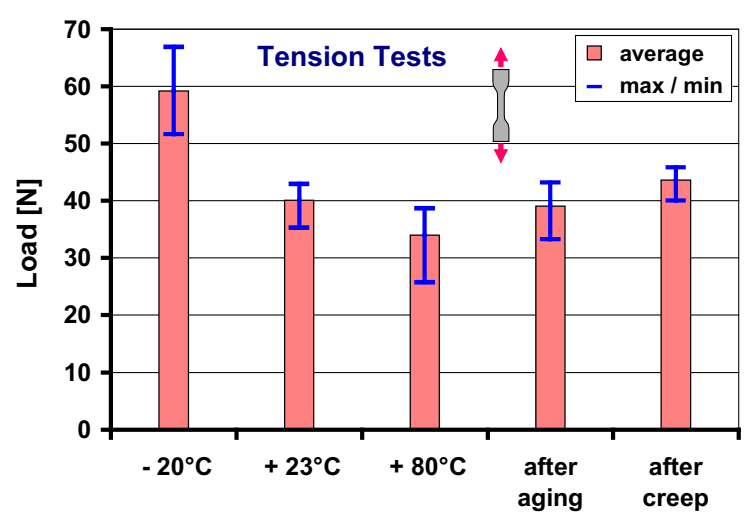

Fig. 32 Dog-bone tension tests under varying conditions

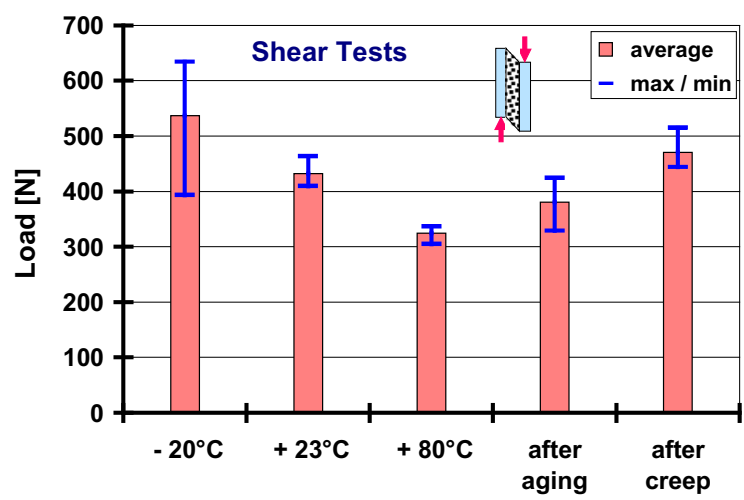

Fig. 33 H-type shear tests under varying conditions

- The results after aging do not significantly differ from unaged specimens for same temperature i.e. room temperature.

- Creep does not degrade the performance comparing the results at room temperature.

- The scatter of results i.e. the bandwidth of loads for all investigated conditions is of comparable size.

Figure 33 displays the related results for shear loading. The results are consistent to those obtained by tension tests. Thus, the mechanisms acting on tension and shear loading are similar and can be extrapolated to more complex load schemes. As only strength values are shown here, it should be added that concerning stiffness, high temperature and aging lead to lower stiffness values according to the load curves of these tests. Thus it is summarized that in view of sizing:

- High temperature is critical in case high strength is required

- Ageing is critical in case high stiffness is required
Regarding aggressive environmental conditions acting on the silicone bonding the design of the bonding might contribute to high durability in addition e.g. by encapsulating the bonding by surrounding PFCs and glass edges with for U-type designs. By this approach, effects of moisture and solar radiation are reduced to a high extent postulating significant benefits in view of durability. In addition, bonding designs shall be favoured of which the free surfaces of the bonding are lowly loaded. The length of the PFC sides might be impacted by this design approach for U-type or L-type bonding designs.

Concluding the statements above, the following approach is suggested in view of complex silicone bondings:

- Establishment of a hyper-elastic material law on the basis of material tests

- Hypothesis for beginning damage indicating service load limitations

- Increased safety factor in regions of relevant environmental impact

As example, point supports might be designed by the following approach for the maximum principal stresses using the properties of the above mentioned two-component structural glazing silicone:

- In general assuming a safety factor of 6 in accordance to the ETAG 002: $2.0 \mathrm{~N} / \mathrm{mm}^{2} / 6=0.33$ $\mathrm{N} / \mathrm{mm}^{2}$

- In the highly encapsulated middle area the safety factor might be lowered to $5: 2.0 \mathrm{~N} / \mathrm{mm}^{2} / 5=$ $0.40 \mathrm{~N} / \mathrm{mm}^{2}$

- In the outboard areas potentially impacted by the aggressive environment, the safety factor might be increased e.g. to $7: 2.0 \mathrm{~N} / \mathrm{mm}^{2} / 7=0.29 \mathrm{~N} / \mathrm{mm}^{2}$

\section{Conclusions and outlook}

Compared to understanding, testing and modelling of conventional material used for structural glazing such as steel and aluminium, no similar basis for silicone as classical bonding material in such kind of applications exist. In order to make the silicone bonding applicable to structural glazing, guidelines were developed such as ETAG 002 placing the design space of structural glazing silicone bonding in a quite tight corset. This corset denies advanced bonding designs to be covered by the guideline and other methodological approaches are required instead. The baseline idea in order to leave 
Fig. 34 Development and test logics based on material and small sample tests

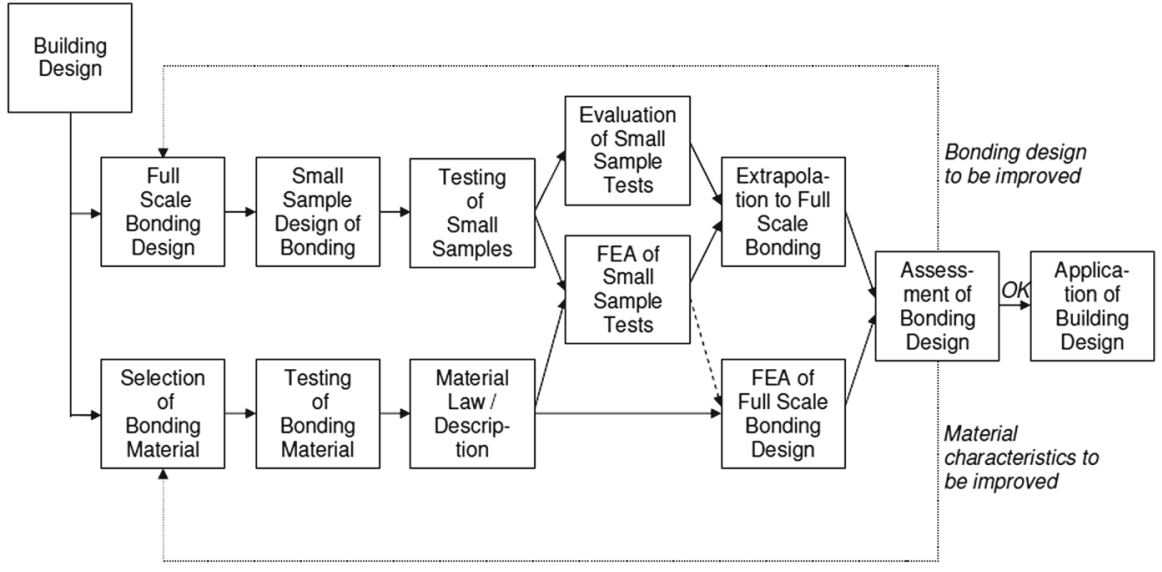

the constraints of guidelines such as ETAG 002 is to develop a comprehensive silicone material model as it exists already for other materials such as steel. It is well understood that such a model is highly complex as different phenomena such as hyper-elasticity, Mullins phenomena and visco-elasticity need to be treated.

In order to step forward in this direction, this paper addresses the full range from silicone material tests characterizing the physical properties of silicone, small sample tests highlighting the impact of boundary conditions on failure mechanisms and complementary structural mechanic analysis to a safety concept for ensuring adequate bonding performance.

Potential pitfalls depicted by this test and development logic exist along several axes:

- In misunderstanding of the behaviour of silicone bondings e.g. the significant stiffening under tension loading evoked by incompressibility in combination with lateral contraction

- In material tests not properly performed due to inadequate clamping of specimens into the testing machine-see Mullin's effect close to load curve origin

- In different failure mechanisms of pure silicone specimens compared to small sample specimens with adequate boundary conditions

- In material laws derived by simple black box approaches of commercial analysis packages and applied beyond the validation ranges.

The paper demonstrates the added value of finite element analysis in view of the small sample tests for understanding the complex behaviour of the bonding especially in view of encapsulation of the silicone material by metal attachments and glass. Obviously limit stress levels exist under tension loading which are almost independent from application design (U-type bonding, point support bonding) for the investigated cases.

Figure 34 presents related development and test logics which can be used for complex bonding geometries with today's knowledge. The assessment of the bonding design based on a synthesis of tests and finite element analyses might indicate that either the silicone material or the bonding design itself is improper asking for an iteration of the development cycle by selecting another material or modifying the design. This loop structure is indicated $b$ the dotted arrows.

Of course, significant efforts still need to be spent towards a comprehensive material model for silicone in view of generality which would allow at least a significant reduction of test efforts. The future roadmap is seen in a safety concept based on partial factors according to EC0 (DIN EN 1990 2002) with special consideration of the hyper-elastic properties of silicon on bondings-work is in progress.

Acknowledgments The author would like to thank Dow Corning GmbH, Germany, and especially Mr. Sigurd Sitte, for comprehensive technical support with respect to the manufacturing of all needed specimens.

\section{Compliance with ethical standards}

Conflict of interest On behalf of all authors, the corresponding author states that there is no conflict of interest. 


\section{References}

DIN 53 504: Prüfung von Kautschuk und ElastomerenBestimmung von Reißfestigkeit, Zugfestigkeit, Reißdehnung und Spannungswerten im Zugversuch (Translation: Testing of rubber-determination of tensile strength at break, tensile stress at yield, elongation at break and stress values in a tensile test) (2009)

DIN EN 1990: EC0 - Basis of structural design. Chapter 6: Verification by the partial factor method: Creation of Load Combinations (2002)

EOTA: ETAG 002—Guideline for European Technical approval for Structural Sealant Glazing systems (SSGS) - Part 1 Supported and unsupported systems (2001)

Fachhochschule München (FHM) FB02: Geklebte Verbindungen im Konstruktiven Glasbau (Translation: Structural bonding in glass constructions) Research Report. BMBF (Federal Ministry of Education and Research) Project, AIF-Nr.: 1755X04 (2007)

Gent, A.N.: Engineering with Rubber-How to Design Rubber Components, 2nd edn. Carl Hanser, München (2001)

Habenicht, G.: Bonding: Basics, Technology, Applications (in German: Original title: Kleben: Grundlagen Technologie Anwendungen ). Springer, Berlin (1997)

Hagl, A.: Ästhetisch, konstengünstig und innovative - Neue Verbindungstechnik für ganz neue tragende Glasstrukturen (Translation: Aesthetic, cost-efficient and innovative - new connection method for brand-new load carrying glass structures). Deutsches Ingenieureblatt (2003)

Hagl, A.: Bemessung von strukturellen Silikonklebungen (Translation: Design of structural silicone bondings). Stahlbau Vol. 76, Issue 8 (2007)

Hagl, A.: Durability by design—new results on load carrying silicone bonding. In: 3rd Symposium on durability of building and construction sealants and adhesives, Denver, CO (2008a)

Hagl, A.: Punktuelles Kleben mit Silikonen (Translation: Pointwise bonding with silicones). Stahlbau Vol. 77, Issue 11, Ernst \& Sohn Verlag, Berlin, Germany (2008b)
Hagl, A.: Silicone bonded point supports - behaviour under cyclic loading, Engineered Transparency-International Conference at glasstec, Düsseldorf, Germany (2010)

Held, B.: Beurteilung von mit Silikonen verklebter Bauteile auf der Basis neuer Erkenntnisse aus der Werkstoffforschung (Translation: Evaluation of bonded building elements based on new findings of materials research). Diplomarbeit, FH München, (2007)

Hess, Dr R. (Glasconsult): 8142 Uitikon, Swizzerland. "Testing of a U-type-bonding as safety devices of the glass panels of various glass Soundbarrier Walls along freeways in the vicinity of Zürich" (2006)

Mullins, L.: Effect of stretching on the properties of rubber. Rubber Chem. Technol. 21(1948), 281-300 (1948)

Pröbster, M.: Elastisch Kleben (Translation: Elastic bondings), Springer Vieweg Wiesbaden, Germany (2013)

Schadow, T.: Beanspruchungsgerechtes Konstruieren von Klebverbindungen in Glastragwerken (Translation: Appropriate construction of adhesive bondings by glass structures), Dissertation, Technische Universität Dresden, (2006)

Treloar, L.R.G.: The Physics of Rubber Elasticity, 3rd edn. Clarendon Press, Oxford (2005)

Wolf, A.T., Descamps, P.: Determination of Poisson's ratio of silicone sealants from ultrasonic and tensile measurements. In: Johnson, P.G. (ed.) Performance of Exterior Building Walls, ASTM STP 1422. American Society for Testing and Materials, West Conshohocken (2002)

Wolf, A.T., Cleland-Host, H.L.: Material properties for use in FEA modeling: sealant behavior with Ambient Laboratory Climate Aging. In: Wolf, A.T. (ed.) Durability of Building and Construction Sealants and Adhesives, ASTM STP 1453. ASTM International, West Conshohocken, PA (2004)

Wolf, A.T.: Preliminary Evaluation of the mechanical properties and durability of transparent structural silicone adhesive (TSSA) for point-fixing in Glazing in preparation for the derivation of static and dynamic design strength values. In: Fourth Symposium on durability and construction sealant and adhesives, June 16-17, Ananheim (CA), (2011) 